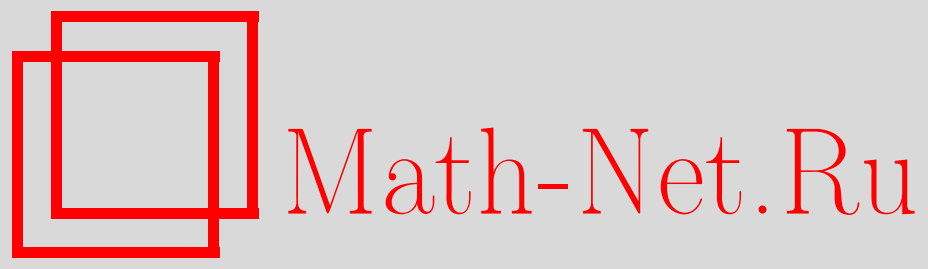

А. Сова, Кольцо Дирихле и безусловные базисы в $L_{2}[0,2 \pi]$, Функи. анализ и его прил., 2013, том 47, выпуск $3,75-81$

DOI: https://doi.org/10.4213/faa3113

Использование Общероссийского математического портала Math$\mathrm{Net.Ru} \mathrm{подразумевает,} \mathrm{что} \mathrm{вы} \mathrm{прочитали} \mathrm{и} \mathrm{согласны} \mathrm{с} \mathrm{пользователь-}$ ским соглашением http://www.mathnet.ru/rus/agreement

Параметры загрузки:

IP : 54.198 .67 .100

26 апреля 2023 г., 14:19:58

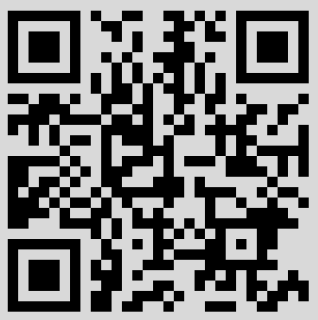


Функционалъный анализ и его приложения

2013, т. 47, вып. 3, с. 75-81

УДК 517.98

\title{
Кольцо Дирихле и безусловные базисы в $L_{2}[0,2 \pi]$
}

\author{
(C) 2013. A. CoBA*
}

Установлено, что кольцо Дирихле допускает представление в бесконечномерной алгебре матриц. Полученные матрицы применяются для построения неортогональных базисов Рисса в сепарабельном гильбертовом пространстве. В таких рамках можно в изобилии строить базисы с интересными свойствами по индивидуальным требованиям. Замечательным образом оказывается, что представление сигналов в любом из этих базисов может быть реализовано численно с помощью быстрых алгоритмов.

В статье обсуждается интересное соответствие между кольцом Дирихле и некоторым классом операторов в гильбертовом пространстве. Устанавливаются также условия, при которых операторы этого специального типа являются линейными гомеоморфизмами. Линейный гомеоморфизм переводит любой ортонормированный базис в базис Рисса, который всегда безусловен ${ }^{1)}$; см., например, [7] или [15].

Основные результаты таковы: теорема 1 характеризует представление рядов Дирихле бесконечными треугольными матрицами. Матрица, полученная таким образом из ряда Дирихле, определяет оператор в гильбертовом пространстве. Теорема 2 дает достаточные условия (на ряд Дирихле и его обратный), при которых этот оператор является линейным гомеоморфизмом, а также двусторонние оценки для получающихся базисов Рисса. Наконец, теорема 3 в явном виде описывает некоторый класс рядов Дирихле, удовлетворяющих использованному в теореме 2 условию. Отметим редкое отличительное свойство построенных здесь базисов: дискретизированные преобразования замены базиса могут быть численно реализованы с помощью быстрого алгоритма сложности $O(N \log N)$ $([12],[13])$.

Множество примеров безусловных базисов возникает при рассмотрении собственных функций линейных дифференциальных операторов (см., например, [11], [6]). Напротив, рассматриваемые в данной заметке безусловные базисы в некоторых случаях связаны с дифференциально-алгебраическими задачами на собственные значения, подобными изученной в [14]; мы останавливаемся на этом более подробно в заключительных замечаниях.

Связь между системами «растянутых» функций, аналогичными системе (2), и рядами Дирихле была подмечена еще в 1945 г. Бёрлингом [3]. Позднее обнаружилось, что свойство базисности Рисса для таких систем можно эквивалентным образом сформулировать в терминах подходящим образом определенных

*Автор выражает благодарность за поддержку Канадскому фонду инноваций (грант LOF \# 22117).

1) Поскольку все ограниченные безусловные базисы суть базисы Рисса, мы отныне будем свободно пользоваться как одним, так и другим термином. 
множителей Дирихле [8]. В отличие от представленных здесь результатов, основанных на явных построениях, такой подход использует аналитические свойства заданных рядами Дирихле функций, которые обычно очень нелегко проверить.

1. Представление рядов Дирихле матрицами. Пусть $A(s)$ - формальный ряд Дирихле вида $A(s)=\sum a_{n} n^{-s}$ (сумма по $n=1,2,3, \ldots$ ), коэффициенты $a_{1}, a_{2}, a_{3}, \ldots$ которого - комплексные числа, а $s$ - комплексная переменная. Хорошо известно, что множество формальных рядов Дирихле образует коммутативное кольцо относительно операций сложения и умножения, заданных формулами $\sum a_{n} n^{-s}+\sum b_{n} n^{-s}=\sum\left(a_{n}+b_{n}\right) n^{-s}$ и $\left(\sum a_{n} n^{-s}\right) \cdot\left(\sum b_{n} n^{-s}\right)=\sum c_{n} n^{-s}$; здесь $c_{n}=\sum_{k l=n} a_{k} b_{l}$, где $k, l$ пробегают все натуральные числа, произведение которых равно $n$. Это кольцо мы называем кольцом Дирихле.

Построим бесконечную матрицу

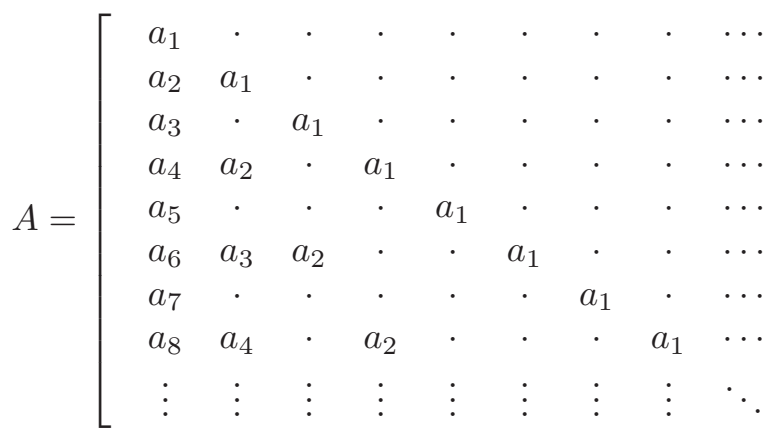

Здесь точками «•» обозначены нулевые элементы. Первый столбец состоит из последовательных коэффициентов $a_{n}$. Во втором столбце элементы той же последовательности расставлены через строку, в третьем - через две и т.Д. Для краткости мы используем обозначение

$$
A=\left[\left(a_{1}, a_{2}, \ldots\right)\right] \text { или просто } A=\left[\left(a_{n}\right)\right] .
$$

Структура матрицы $A$ напоминает решето Эратосфена. Заметим, что $I=$ $[(1,0,0, \ldots)]$. Для сравнения, матрица $Z=[(1,1,1, \ldots)]$ соответствует ряду, задающему дзета-функцию Римана $\zeta(s)=\sum n^{-s}$. Число элементов $a_{k}$ в $n$-й строке есть в точности число $d(n)$ делителей числа $n$. Следующее наблюдение является ключевым для наших рассуждений.

Теорема 1. Если

$$
A(s)=\sum a_{n} n^{-s}, \quad B(s)=\sum b_{n} n^{-s},
$$

mo

$$
A(s) B(s)=\sum c_{n} n^{-s} \text { тогда и только тогда, когда }\left[\left(a_{n}\right)\right] \cdot\left[\left(b_{n}\right)\right]=\left[\left(c_{n}\right)\right] .
$$

Если, кроме того, $a_{1} \neq 0$, то

$$
\frac{1}{A(s)}=\sum d_{n} n^{-s} \quad \text { тогда и только тогда, когда }\left[\left(a_{n}\right)\right]^{-1}=\left[\left(d_{n}\right)\right] .
$$

Доказательство. Обе эквивалентности проверяются прямой выкладкой. Коэффициенты произведения $A(s) B(s)$ даются формулой $c_{n}=\sum_{k \mid n} a_{k} b_{n / k}$. Далее, у $A(s)$ имеется обратный тогда и только тогда, когда $a_{1} \neq 0$. В таком случае 
коэффициенты $\left(d_{n}\right)$ ряда $A(s)^{-1}$ удовлетворяют соотношению $\sum_{k \mid n} a_{k} d_{n / k}=$ $\delta_{n, 0}$.

Замечание 1. Предложенное отождествление ряда Дирихле с матрицей - не единственный способ добиться такого результата. В качестве другого варианта можно положить $A=\left[\left(a_{1}, a_{2} 2^{-s}, a_{3} 3^{-s}, \ldots\right)\right]$, где $s-$ переменная или фиксированное число. Выбор $s=0$ упрощает формулы. Можно также использовать матрицы, транспонированные к указанным выше, чтобы получить альтернативное (двойственное) представление кольца Дирихле.

Замечание 2. Отметим также, что конечные вычисления с матрицами, усеченными до левой верхней подматрицы размера $N \times N$, дают в этом интервале точные результаты. Поскольку в $n$-й строке имеется $d(n)$ нетривиальных коэффициентов, требуется $d(1)+\cdots+d(N)=O(N \log N)$ нетривиальных сложений и умножений, чтобы вычислить значение матрицы на векторе ${ }^{1)}$. На самом деле это можно сделать, используя алгоритм, исключающий необходимость хранить тривиальные и избыточные элементы матрицы [13].

Построение базисов в $\boldsymbol{L}_{\mathbf{2}}[\mathbf{0}, \mathbf{2} \boldsymbol{\pi}]$. Пусть $e_{n}=e^{i n t} / \sqrt{2 \pi}$, так что $\left\{e_{n}\right\}_{-\infty}^{\infty}$ - ортонормированный базис в $L^{2}[0,2 \pi]$. Далее, пусть $L^{2}[0,2 \pi]=H_{a} \oplus H_{h}-$ разложение пространства $L^{2}[0,2 \pi]$ в прямую сумму двух подпространств, отвечающих неположительным и положительным частотам соответственно. В подпространстве $H_{h}$ рассмотрим семейство функций, определяемых суммируемой с квадратом последовательностью комплексных чисел $\left(\alpha_{l}\right)_{l=1}^{\infty}$ следующим образом:

$$
f_{m}(t)=\sum_{l=1}^{\infty} \alpha_{l} \frac{e^{i m l t}}{\sqrt{2 \pi}}=\sum_{l=1}^{\infty} \alpha_{l} e_{m l}, \quad m=1,2,3, \ldots
$$

Обратим внимание на масштабирование $f_{m}(t)=f_{1}(m t)$. Kроме того, у всех $f_{m}$ одна и та же норма, $\left\|f_{m}\right\|=\left(\sum\left|\alpha_{l}\right|^{2}\right)^{1 / 2}<\infty$. На протяжении всей статьи нам будет нужна только сходимость по норме. Заметим, однако, что в силу теоремы Карлесона [4] ряд для $f_{m}$ сходится почти всюду.

Нам хотелось бы понять основные свойства системы $\left\{f_{m}\right\}_{m=1}^{\infty} \subset H_{h}$ как возможного базиса. Используя (2), вычислим

$$
\phi(t)=\sum_{m=1}^{\infty} x_{m} f_{m}(t)=\sum_{n=1}^{\infty} \sum_{k \mid n} \alpha_{k} x_{n / k} e_{n}=\sum_{n=1}^{\infty} y_{n} e_{n} .
$$

Таким образом, коэффициенты Фурье $y_{n}$ связаны с $x_{m}$ формулой

$$
\left[y_{1}, y_{2}, \ldots\right]^{\prime}=T\left[x_{1}, x_{2}, \ldots\right]^{\prime}, \quad T=\left[\left(\alpha_{n}\right)\right]
$$

где «штрих» обозначает транспонирование. По теореме 1

$$
T^{-1}=\left[\left(\gamma_{n}\right)\right]=\left[\left(\alpha_{n}\right)\right]^{-1},
$$

где $\left(\gamma_{n}\right)$ - последовательность, обратная по Дирихле к $\left(\alpha_{n}\right)$.

1) Известны гораздо более точные оценки для суммы числа делителей; см., например, [1] или [5]. Впервые оценки такого типа были получены Дирихле в первой половине XIX века. 
Далее, определим $g_{n}(t)$ как функцию, коэффициенты Фурье которой суть числа, комплексно-сопряженные к элементам $n$-й строки матрицы $T^{-1}$, т. е.

$$
g_{n}(t)=\sum_{k \mid n} \bar{\gamma}_{n / k} e_{k}
$$

или, что то же самое, $g_{n}=\left(T^{*}\right)^{-1} e_{n}$. Сразу ясно, что

$$
\left\langle f_{m} \mid g_{n}\right\rangle=\delta_{m, n} .
$$

Заметим, что $g_{n} \in H_{h}$ - тригонометрические многочлены.

Обратимся теперь к анализу и укажем необходимое условие для того, чтобы отображение $T$ было гомеоморфизмом:

Теорема 2. Если и последовательность $\left(\alpha_{l}\right)$, и ее обратнал по Дирихле $\left(\gamma_{l}\right)$ абсолютно суммируемы (т.е. лежат в $\left.l_{1}\right)$, то справедливы следующие утверждения.

А. Отображсение T, определенное в (3), - линейный гомеоморфизм. В частности, система $\left\{f_{m}\right\}$, заданная формулой (2), является базисом Рисса в $H_{h}$. Биортогональная система $\left\{g_{n}\right\}$ - также базис Рисса. Более того, для произвольного элемента $\phi \in H_{h}$ имеет место представление

$$
\phi=\sum_{n=1}^{\infty}\left\langle\phi \mid g_{n}\right\rangle f_{n} .
$$

В. Пусть $A=\left(\sum_{l}\left|\alpha_{l}\right|\right)^{-2}, B=\left(\sum_{l}\left|\gamma_{l}\right|\right)^{2}$. Тогда для произвольного элемента $\phi \in H_{h}$ выполнена двусторонняя оценка

$$
A\|\phi\|^{2} \leqslant \sum_{n=1}^{\infty}\left|\left\langle\phi \mid g_{n}\right\rangle\right|^{2} \leqslant B\|\phi\|^{2} .
$$

Доказательство. А. Хорошо известно, что если для действующего в гильбертовом пространстве оператора $l_{1}$-нормы всех столбцов и строк его матрицы в некотором ортонормированном базисе равномерно ограничены, то оператор ограничен (доказательство см. в [9, пример 2.3 гл. III]). Заметим, что $l_{1}$-норма каждого столбца матрицы $T$ равна $M=\sum_{n=1}^{\infty}\left|\alpha_{n}\right|<\infty$, а $l_{1}$-норма каждой ее строки заведомо ограничена сверху числом $M$. Таким образом, оператор $T$ непрерывен. Аналогично, из условия $\left(\gamma_{l}\right) \in l_{1}$ вытекает, что оператор $T^{-1}$ непрерывен. Итак, $T$ - линейный гомеоморфизм. Так как $f_{m}=T e_{m}$, то $\left\{f_{m}\right\}$ - базис Рисса. Поэтому любую функцию $\phi \in H_{h}$ можно единственным образом представить в виде $\phi=\sum_{m=1}^{\infty} x_{m} f_{m}$. Из формулы (4) следует, что $x_{n}=\left\langle\phi \mid g_{n}\right\rangle$, что доказывает соотношение (5). Хорошо известно также, что базис, биортогональный к базису Рисса, автоматически является базисом Рисса (см., например, $[7$, гл. VI]).

В. Для краткости положим $x_{n}=\left\langle\phi \mid g_{n}\right\rangle$ и $y_{k}=\left\langle\phi \mid e_{k}\right\rangle$, так что $\sum\left|y_{k}\right|^{2}=\|\phi\|^{2}$. Тогда

$$
\left|\sum_{k \mid n} \gamma_{n / k} y_{k}\right|^{2} \leqslant\left(\sum_{k \mid n}\left|\gamma_{n / k}\right|^{1 / 2}\left|\gamma_{n / k}\right|^{1 / 2}\left|y_{k}\right|\right)^{2} \leqslant\left(\sum_{k \mid n}\left|\gamma_{n / k}\right|\right) \sum_{k \mid n}\left|\gamma_{n / k}\right|\left|y_{k}\right|^{2}
$$


и, тем более,

$$
\left|\sum_{k \mid n} \gamma_{n / k} y_{k}\right|^{2} \leqslant \sum_{l}\left|\gamma_{l}\right| \sum_{k \mid n}\left|\gamma_{n / k}\right|\left|y_{k}\right|^{2}
$$

Отсюда следует, что

$$
\sum_{n}\left|x_{n}\right|^{2}=\sum_{n}\left|\sum_{k \mid n} \gamma_{n / k} y_{k}\right|^{2} \leqslant \sum_{l}\left|\gamma_{l}\right| \sum_{n} \sum_{k \mid n}\left|\gamma_{n / k}\right|\left|y_{k}\right|^{2} .
$$

Перегруппировка слагаемых $\sum_{n} \sum_{k \mid n}\left|\gamma_{n / k}\right|\left|y_{k}\right|^{2}=\sum_{l}\left|\gamma_{l}\right| \sum_{n}\left|y_{n}\right|^{2}$ в последней двойной сумме приводит к соотношению

$$
\sum_{n}\left|x_{n}\right|^{2} \leqslant\left(\sum_{l}\left|\gamma_{l}\right|\right)^{2} \sum_{n}\left|y_{n}\right|^{2}
$$

т. е. к правому неравенству в формуле (6). Левое неравенство получается, если в приведенных выше оценках поменять $x_{n}$ и $y_{n}$ ролями и заменить $\gamma_{n}$ на $\alpha_{n}$.

Замечание 1. Хорошо известны примеры условных базисов (см., например, [2], [10]).

Замечание 2. Как отмечено выше, вычисление значения матрицы (1) размера $N \times N$ на векторе требует выполнения $O(N \log N)$ нетривиальных умножений и сложений. В сочетании с быстрым преобразованием Фурье это дает быстрый и эффективный алгоритм сложности $O(N \log N)$ для представления векторов в базисах $\left\{f_{m}\right\}$ типа $(2)$. Одной из областей применения такого алгоритма является анализ электронных сигналов [12].

По-видимому, не известно ни одного универсального критерия, который бы характеризовал последовательности из $l_{1}$, для которых обратная по Дирихле последовательность также лежит в $l_{1}$. Теорема 3 ниже дает некоторое достаточное условие. Она основана на следующем наблюдении.

Лемма. Для любой положительной постоянной $c \leqslant 1 /\left(2 \cdot 3^{4 / 3}+3^{2 / 3}\right) \simeq$ 0,0932 и любого $n \geqslant 2$ выполнено неравенство

$$
\sum_{d \mid n, d \geqslant 2} c^{d} d^{2} \leqslant \frac{c}{2}
$$

Доказательство. Пусть $a=3^{3 / 2} \simeq 2,081$. Легко видеть, что $d^{2} \leqslant a^{d}$ для всех целых $d \geqslant 2$. При $0<c<1 / a$ имеем

$$
\sum_{d \mid n, d \geqslant 2} c^{d} d^{2} \leqslant \sum_{d \mid n, d \geqslant 2} c^{d} a^{d} \leqslant(a c)^{2}\left[1+(a c)+(a c)^{2}+\cdots\right]=\frac{(a c)^{2}}{1-a c} .
$$

Заметим, что правая часть не превосходит $c / 2$ при $c \leqslant 1 /\left(2 a^{2}+a\right)$.

Теорема 3. Пусть $\left|\alpha_{1}\right| \geqslant c / 2 u\left|\alpha_{n}\right| \leqslant c^{n}$ при $n \geqslant 2$ с некоторой постоянной $c \leqslant 1 /\left(2 \cdot 3^{4 / 3}+3^{2 / 3}\right)$. Тогда

$$
\sum_{l}\left|\alpha_{l}\right|<\infty, \quad \sum_{l}\left|\gamma_{l}\right|<\infty,
$$

где $\left(\gamma_{n}\right)$ - последовательность, обратная по Дирихле $к$ последовательности $\left(\alpha_{n}\right)$. 
Доказательство. Поскольку по условию теоремы модули $\left|\alpha_{n}\right|$ экспоненциально убывают, последовательность суммируема. Чтобы доказать, что последовательность $\left(\gamma_{n}\right)$ также абсолютно суммируема, воспользуемся рекуррентной формулой для обратной по Дирихле последовательности (см., например, [1, теорема 2.8]). А именно, $\gamma_{1}=1 / \alpha_{1}$ и

$$
\gamma_{n}=-\frac{1}{\alpha_{1}} \sum_{d \mid n, d>1} \alpha_{d} \gamma_{n / d} \quad(n \geqslant 2) .
$$

Докажем по индукции, что

$$
\left|\gamma_{k}\right| \leqslant \frac{2}{c} \frac{1}{k^{2}} \quad \text { для всех } k .
$$

Так как $\gamma_{1}=1 /\left|\alpha_{1}\right|$, то это неравенство выполнено при $k=1$. Предполагая, что оно выполнено для всех $k=1, \ldots, n-1$, получим из (7) и леммы 1 , что

$$
\begin{aligned}
\left|\gamma_{n}\right| & \leqslant \frac{2}{c} \sum_{d \mid n, d>1} c^{d}\left|\gamma_{n / d}\right| \leqslant \frac{2}{c} \sum_{d \mid n, d>1} c^{d} \frac{2}{c}\left(\frac{d}{n}\right)^{2} \\
& =\frac{4}{c^{2}} \frac{1}{n^{2}} \sum_{d \mid n, d>1} c^{d} d^{2} \leqslant \frac{4}{c^{2}} \frac{c}{2} \frac{1}{n^{2}}=\frac{2}{c} \frac{1}{n^{2}} .
\end{aligned}
$$

Тем самым (8) доказано. Значит, $\sum_{l}\left|\gamma_{l}\right|<\frac{2}{c} \sum_{l} \frac{1}{l^{2}}<\infty$.

Замечание 3. Интересно было бы выяснить, действительно ли $\gamma_{n}$ обязаны убывать экспоненциально. Хотя ответ и неизвестен, численные эксперименты подсказывают, что он должен быть отрицательным.

Замечание 4. Частный случай базисов типа, рассматриваемого в данной работе, возникает в нелинейных задачах на собственные значения. Как показано в [14], уравнение

$$
\mu y^{\prime \prime}(t)=y(t)-\frac{1}{y(t)}, \quad t \in[0,2 \pi) \simeq S^{1},
$$

допускает решение вида $y=1+f(t)$, где $f \in H_{h}$, тогда и только тогда, когда $\mu=\mu_{m}=-2 / m^{2}(m=1,2, \ldots)$. Соответствующие собственные функции имеют вид

$$
y_{m}(t)=1+f_{m}(t)=1+\sum_{l=1}^{\infty} \alpha_{l} e^{i m l t},
$$

где коэффициенты $\alpha_{n}$ определяются рекуррентными формулами

$$
\alpha_{1}=a, \quad \alpha_{l}=-\frac{1}{2\left(l^{2}-1\right)} \sum_{r=1}^{l-1} \alpha_{l-r} \alpha_{r}\left(2 r^{2}-1\right), \quad l \geqslant 2 .
$$

Здесь $a-$ свободный (вещественный) параметр. Легко видеть, что коэффициенты $\alpha_{l}$ ведут себя как $a^{l}$ и для достаточно малых $a$ удовлетворяют условия теоремы 3. Поэтому для подходящим образом выбранного параметра $a$ последовательность $\left\{f_{m}\right\}$ является базисом Рисса в $H_{h}$. 
Благодарности. Я хотел бы поблагодарить доктора М. Чугунову за интересную дискуссию в ходе выполнения этой работы. Я также благодарен рецензентам, указавшим на многочисленные способы улучшить эту статью. В частности, один из них обнаружил дыру в доказательстве и предложил заделать ее кратким рассуждением, из которого выросла часть А теоремы 2. Так что заслугу установления этой теоремs я должен разделить с упомянутым рецензентом. Я в долгу и перед другим рецензентом, который предложил улучшения в некоторых оценках, а также в теореме 3 .

\section{Литература}

[1] T. M. Apostol, Introduction to Analytic Number Theory, Springer-Verlag, New YorkHeidelberg-Berlin, 1976.

[2] К. И. Бабенко, О сопряженных функииях, Докл. АН СССР, 62:2 (1948), 157-160.

[3] A. Beurling, The Collected Works of Arno Beurling, in: Harmonic Analysis, Contemp. Math., vol. 2, Birkhäuser, Boston, 1989, 378-380.

[4] L. Carleson, On convergence and growth of partial sums of Fourier series, Acta Math., 116 (1966), 135-157.

[5] K. Chandrasekharan, Arithmetical Functions, Springer-Verlag, New York-HeidelbergBerlin, 1970.

[6] P. Djakov, B. Mityagin, Bari-Markus property for Riesz projections of $1 D$ periodic Dirac operators, Math. Nachr., 283 (2010), 443-462.

[7] И. Ц. Гохберг, М. Г. Крейн, Введение в теорию линейных несамосопряженных операторов в гилъбертовом пространстве, Наука, М., 1965.

[8] H. Hedenmalm, P. Lindqvist, K. Seip, A Hilbert Space of Dirichlet Series and Systems of Dialated Funcitons in $L^{2}(0,1)$, Duke Math. J., 86 (1997), 1-37.

[9] Т. Като, Теория возмущений линейных операторов, Мир, М., 1972.

[10] А. М. Олевский, Об операторах, производдщих условные базисы в гильбертовом пространстве, Матем. заметки, 12:1 (1972), 73-84.

[11] А. А. Шкаликов, О базисности собственных функиий обыкновенного дифференииального оператора, УМН, 34:5(209) (1979), 235-236.

[12] A. Sowa, A fast-transform basis with hysteretic features, in: IEEE Conference Proceedings: Electrical and Computer Engineering (CCECE), 2011 24th Canadian Conference on, 8-11 May 2011, 000253-000257.

[13] A. Sowa, Factorizing matrices by Dirichlet multiplication, Linear Algebra Appl., 438:5 (2013), 2385-2393.

[14] A. Sowa, On an eigenvalue problem with a reciprocal-linear term, Waves in Random and Complex Media, 22 (2012), 186-206.

[15] R. M. Young, An Introduction to Nonharmonic Fourier Series, Academic Press, New York-London-Toronto, 1980.

Department of Mathematics and Statistics

University of Saskatchewan, Canada

Поступило в редакцию

e-mail: sowa@math.usask.ca

6 июня 2011 г. 